\title{
Lung Shunt Dose With A Planar Versus Spect-CT Images In Planification Of HCC Treatment
}

\author{
Raquel Barquero ( $\square$ rbarquerosa@saludcastillayleon.es ) \\ Hospital Clínico Universitario de Valladolid: Hospital Clinico Universitario de Valladolid \\ https://orcid.org/0000-0002-3324-5886 \\ Javier Velasco \\ Valladolid University Hospital: Hospital Clinico Universitario de Valladolid \\ Antonio Hurtado \\ Valladolid University Hospital: Hospital Clinico Universitario de Valladolid \\ Berta Pérez \\ Valladolid University Hospital: Hospital Clinico Universitario de Valladolid \\ Ricardo Ruano \\ Valladolid University Hospital: Hospital Clinico Universitario de Valladolid
}

Original research

Keywords: Hepatic tumor, SIRT 90Y, treatment planification, dosimetry, nuclear medicine, radioembolization, yttrium

Posted Date: May 5th, 2021

DOl: https://doi.org/10.21203/rs.3.rs-455117/v1

License: (c) (1) This work is licensed under a Creative Commons Attribution 4.0 International License.

Read Full License 


\section{LUNG SHUNT DOSE WITH A PLANAR VERSUS SPECT-CT IMAGES IN PLANIFICATION OF HCC TREATMENT}

Raquel Barquero ${ }^{1}$, Javier Velasco ${ }^{2}$, Antonio Hurtado ${ }^{3}$, Berta Pérez ${ }^{4}$, Ricardo Ruano

1 Hospital Clinico Universitario Valladolid, SPAIN, rbarquerosa@saludcastillayleon.es

2 Hospital Clinico Universitario Valladolid, SPAIN, jvelasco@saludcastillayleon.es

3 Hospital Clinico Universitario Valladolid, SPAIN, ahurtado@ saludcastillayleon.es

4 Hospital Clinico Universitario Valladolid, SPAIN, bertaperez@saludcastillayleon.es

5 Hospital Clinico Universitario Valladolid, SPAIN, rruano@saludcastillayleon.es

\section{ABSTRACT}

PURPOSE: The purpose is to investigate the possibility of evaluate the lung shunt fraction $L S F$ with SPECT image in planning with Tc-99m of hepatic HCC treatments with Y-90 microspheres, comparing the results with those obtained with planar images. METHODS: Firstly, for a patient with a predictably high shunt, the 4 planar images are used as in the classic method to determine PLANAR LSF. Second, to determine the SPECT $L S F$, the SPECT images acquired for planning the therapeutic activity, and therefore centered in the hepatic region, are used. These images does not cover the entire lung but only some slices. Advantage is taken of the fact that the distribution of ${ }^{99 \mathrm{M}} \mathrm{Tc}$ in healthy lung tissue is homogeneous. Then the activity concentration in the lung can be known and the SPECT $L S F$ can be calculated. RESULTS: The values obtained for PLANAR $L S F$ and SPECT $L S F$ are $11 \%$ and $28 \%$, respectively. The results for the lung dose $D_{\text {lung }}$ are of $24.50 \mathrm{~Gy}$ and $7.92 \mathrm{~Gy}$, respectively. CONCLUSION: In this study, $L S F$ and lung absorbed dose $D_{\text {lung }}$ based in planar static and SPECT/CT images acquired with ${ }^{99 \mathrm{M}} \mathrm{Tc}$, have been described and compared. The results show a dependence between the absorbed dose in the lung with the $L S F$ according to a rational function $f=L S F /(1-L S F)$. The values of $L S F$ and $D_{\text {lung }}$ obtained with planar method are 2.51 and 3.09 higher than the values obtained with SPECT method, respectively. This is attributed to the differences in the quantification method, especially to the absence of attenuation correction in the planar method. In the $L S F$ values, the difference is direct, but in the case of the $D_{\text {lung }}$ the difference is greater due to covariant dependence of dose with activity and LSF. Practically for the future, the SPECT-CT method is better and will be applied in new patients.

\section{KEYWORDS}

Hepatic tumor, SIRT 90Y, treatment planification, dosimetry, nuclear medicine, radioembolization, yttrium

\section{DECLARATION}

Ethics approval and consent to participate:

- All patients have signed the corresponding informed consent 
- The ethical committee that approves the study is the Ethics Committee of the Valladolid clinical university hospital, Spain.

\section{Consent for publication}

Not applicable

\section{$\underline{\text { Availability of data and material }}$}

Data sharing is not applicable to this article as no datasets were generated or analyzed during the current study.

\section{Competing interests}

The author(s) declare(s) that they have no competing interests.

\section{Funding}

There are no sources of funding for the study.

\section{$\underline{\text { Authors' contributions }}$}

Conception and methodological design: Raquel Barquero, Javier Velasco and Antonio Hurtado. Clinical analysis of images: Javier Velasco, Berta Perez y Ricardo Ruano. Cuantitative analysis of images: Raquel Barquero and Antonio Hurtado.

Acknowledgements

No author to mention specially.

\section{PURPOSE}

Perfusion of $90 \mathrm{Y} \mu$-spheres for the treatment of liver malignancies with trans-arterial radio embolization (TARE) has been used extensively since the last ten years, with first reports of $90 \mathrm{Y}$ radioembolization in hepatic malignancies in the 1960s. In 1999 the FDA approved a humanitarian device exemption for the use of glass microspheres in patients with unresectable HCC, and in 2002 for resin microspheres for colorectal cancer liver metastases [1]. Activities of 1-3 GBq are usually perfused and a number of studies has been developed with the outcome of the treatment. One of the problem to undergoing the treatment is the development of hepatopulmonary shunting in hepatic tumors. To evaluate if it is possible to go ahead with the lung risk toxicity, and before the treatment, the lung shunt fraction $(L S F)$ with MAA-99MTc is investigated leading a wide range of published $L S F$ values [2, 3, 4].

Usually [5] the $L S F$ values reported are based in 4 planar image acquired with Gammacamera (GC). Even though these planar images only take 20 minutes (10 min per acquisition in a GC with two heads), SPECT CT imaging is now widely available and is always used to quantify perfused and tumor volumes and activities in the patient 
liver, therefore centered in the hepatic region. Although these images does not cover the entire lung, can be used to LSF determination. The aim of this study is to investigate the possible differences between the two methods of determining $L S F$, with PLANAR image and with those slices of the SPECT image that cover the lung. The corresponding $L S F$ values at each approach are obtained in the lungs regions corresponding to one patient with high $L S F$. The lung absorbed dose dependence with $L S F$ is also investigated.

\section{METHODS}

\section{A. Patients}

The patients selected for ${ }^{90}$ Ytrium-microshere hepatic radioembolization (TARE) with LTC Essen algorithm [4] undergoing a pre-treatment planning with MAA- ${ }^{99 \mathrm{M}} \mathrm{Tc}$ spheres $(19 \pm 6)$ days before the treatment with ${ }^{90} \mathrm{Y}$.

In this study one patient with high shunt fraction has been selected. An activity of 222 $\mathrm{MBq}$ of $\mathrm{MAA}^{99 \mathrm{M}} \mathrm{Tc}$ diluted in $5 \mathrm{ml}$ of physiological serum within a syringe and measured in a calibrated Capintec-512 [6] is prepared for the infusion. In a vascular room, the interventional radiologist defined the arterial way to reach to the tumor and prepared the infusion catheter. After, the Nuclear Medicine physician (NMP) introduced the radiopharmaceutical in the vascular pre-defined way. Lastly, the patient is transferred to Nuclear Medicine Department to acquire the needed images.

\section{B. Lung Shunt Fraction \\ 1. Method 1: Planar acquisitions}

Two static scan planar imaging were acquire after 99MTc perfusion, one centered in the lung region and the other centered in the liver region, both of them with two acquisition views, $0^{\circ}(\mathrm{AP})$ and $180^{\circ}$ (PA) for each patient. The time of each acquisition was 10 minutes with a dual-head Siemens SYMBIA T2 gamma camera with a crystal thickness of $9.5 \mathrm{~mm}$ equipped with Low-Energy High Resolution collimators, LEHR. SYNGO station was used by NMP to draw the lung and liver ROI's (see Figure 1): 


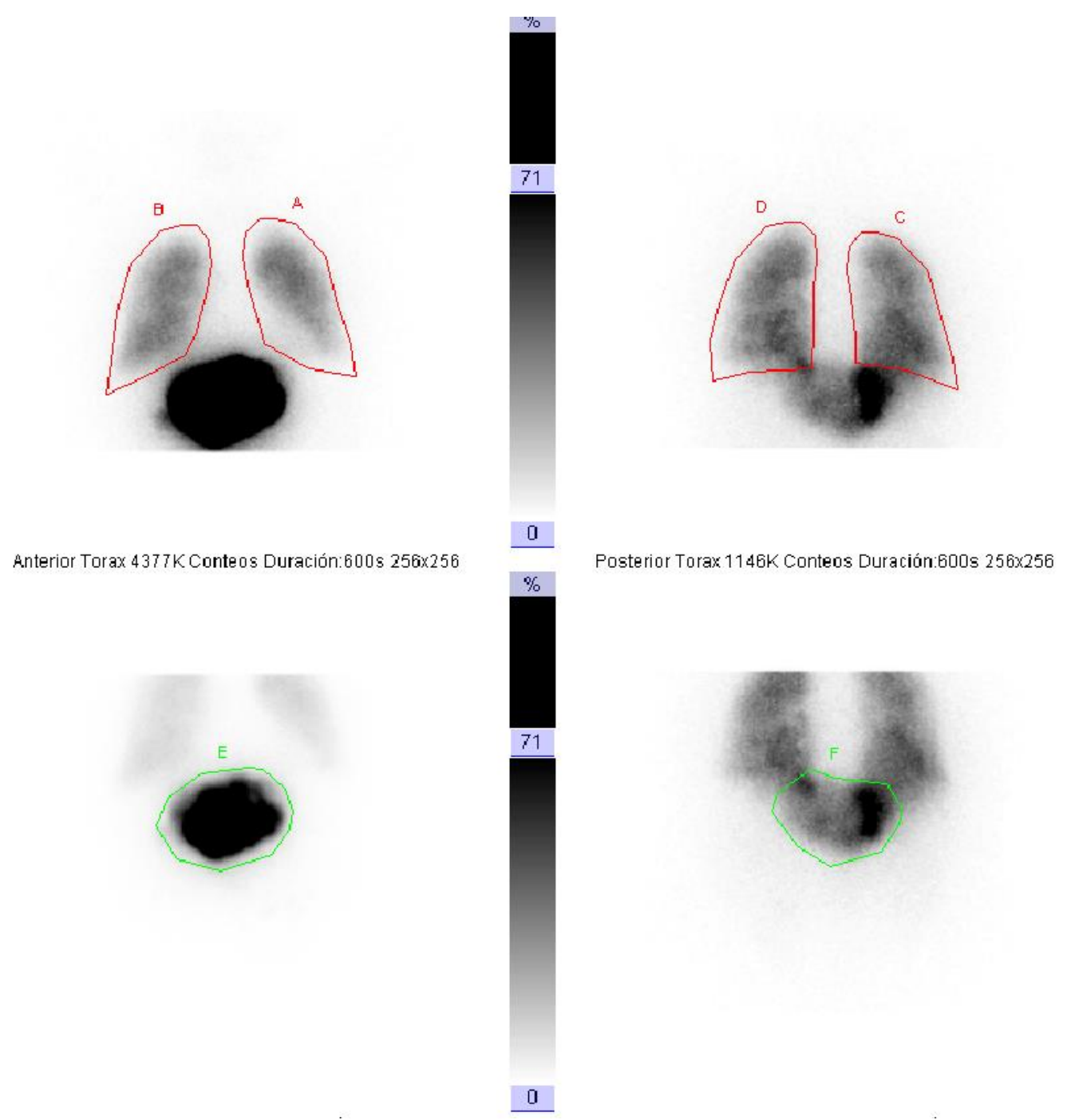

Fig.1. ROI of lung and liver of one patient. Images show, from top to bottom, lung ROIS and liver ROIS. From right to left the $0^{\circ}$ image and $180^{\circ}$ image, respectively. Two static acquisitions are needed to complete the lungs and the liver as a whole regions.

The lung shunt fraction $L S F$ percent was calculated as the following [7]:

$$
L S F=\frac{C_{\text {lung }}}{C_{\text {lung }}+C_{\text {liver }}} \times 100
$$

Where, see figure 1:

$L S F$ was the lung shunt fraction as a percentage

$C_{\text {lung }}$ was the activity, in counts, of the lung segmented regions right and left (sum of B, A, D and C in Figure 1), 
$C_{\text {liver }}$ was the activity, in counts, of the segmented liver region (sum of $\mathrm{F}$ and $\mathrm{E}$ in Figure 1).

\section{SPECT-CT acquisitions (Method 2)}

To calculate the activity in the perfused liver tissue PV and Tumor liver tissue T counts and volumes inside the liver a SPECT/CT acquisition centered in the liver region was performed for each patient. SPECT/CT acquisitions was preformed employing the SYMBIA T2 gamma camera: SPECT acquired with 32 frames, 30 s/frame (960 s), 128 $\times 128$ matrix and $4.8 \mathrm{~mm}$ voxel. CT parameters are: $110 \mathrm{kVp}, 63 \mathrm{mAs}, 75 \mathrm{rpm}$. Reconstruction was done with Siemens Flash3D ${ }^{\mathrm{TM}}$, OS-EM, 8 iterations 4 subsets, CDR compensation, CT-derived Attenuation Correction and energy window-based Scatter Correction. As the collimator used was a LEHR, the sensitivity $\varepsilon$ was $92 \mathrm{cps} / \mathrm{MBq}$ (per detector) [8].

Using the slices of the lung region of this SPECT CT image enclosed in PV and T liver tissue the estimation the $L S F$ and the corresponding lung tissue dose was done.

Analysis of images was performed using ImageJ software [9].

The total activity in the patient was estimated from the total counts in all the reconstructed slices $N, C_{\text {total }}$ :

$$
C_{\text {total }}=\sum_{i=1}^{N} C_{i}
$$

Where,

$\mathrm{C}_{\mathrm{i}}$ was the total counts in slice $\mathrm{i}$.

Total number of lung counts $x$ to $N C_{\text {lungs }}$ was estimated from the average of the activity concentration in the slices covered by the lung in the SPECT-CT $c$, (in Figure 2 an example of slice in the SPECT/CT image for the patient can be seen):

$$
C=\frac{\sum_{i=x}^{N} \overline{c_{l}}}{2 \times(N-x)}
$$

Where:

$c_{i}$ are the mean counts $(\mathrm{c} / \mathrm{ml})$ in each ROI drawn in each slice within lungs region.

$x$ was the first slice and $N$ was the last slice where lungs appears. In Figure 2 an example of slice in the SPECT/CT image in which the lungs of one patient can be seen.

$N-x$ are the number of slices with lung regions and $2 *(N-x)$ are the number of ROI's drawn (2 per slice). In each slice from $x$ to $N$, ROI's were determined drawing a VOI around lung obtained from the CT images [10]. 
Based on $0.3 \mathrm{~g} / \mathrm{ml}$ as lung tissue density $[11,12]$ and a lung tissue mass $M_{\text {lung }}$ of $\sim 1000$ $\mathrm{g}[6,13]$, the total lung count $C_{\text {lung }}$ was determined as:

$$
C_{\text {lung }}=c \times \frac{1000}{0.3}
$$

Then, the $L S F$ can be calculated from equations 2 and 4, as:

$$
L S F=\frac{C_{\text {lung }}}{C_{\text {total }}} \times 100
$$

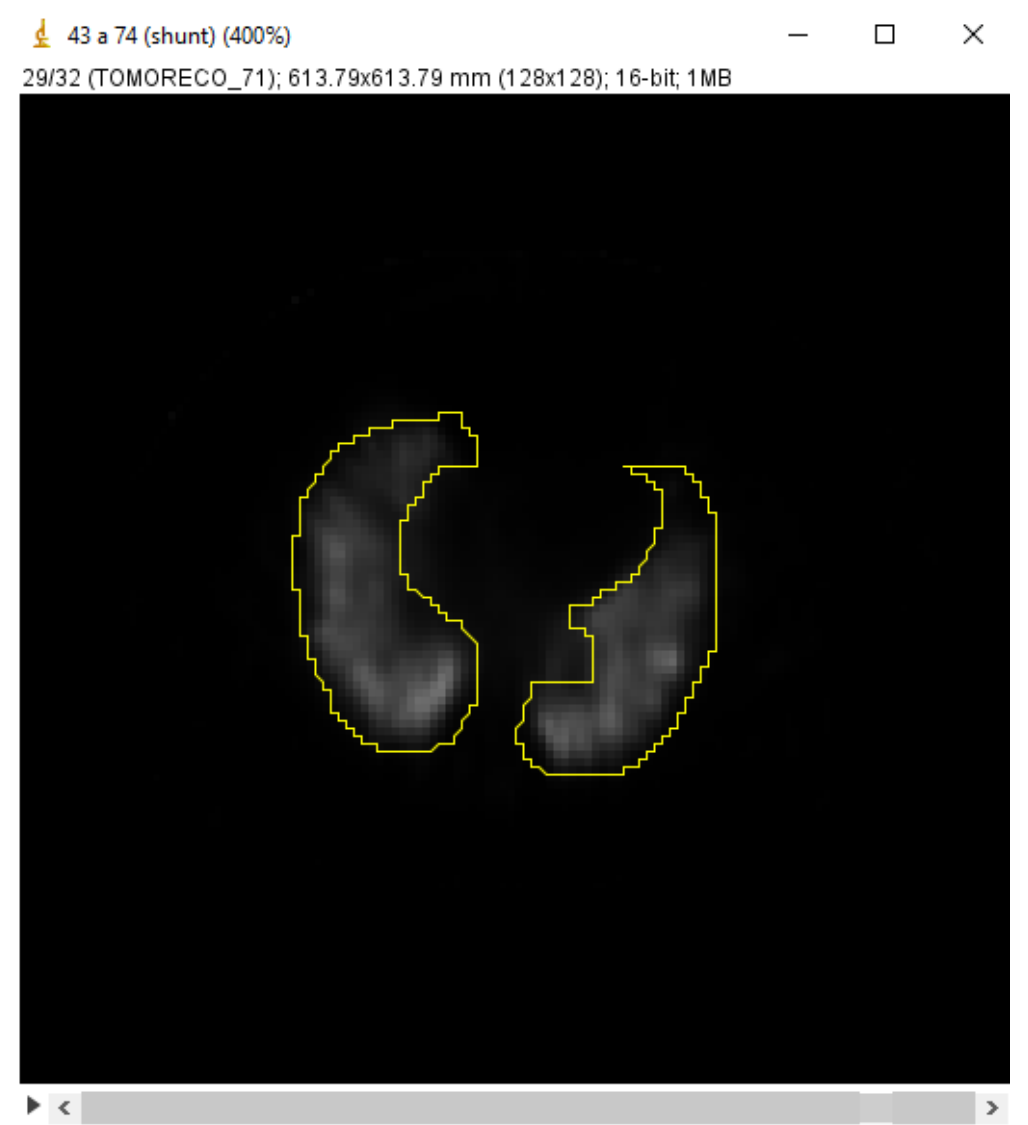

Fig.2. Reconstructed TRANSAXIAL SPECT image of one slice for one patient (LEHR collimation, $4.7295 \mathrm{~mm}$ voxel, $30 \mathrm{~ms}$ actual frame duration, 32 frames in rotation, OSEM 8i4s). VOIs were drawn manually using CT boundaries as reference.

Figure 3 is the reconstruction with MATLAB [14] of the slices $x$ to $N$. The upper area of the image in figure 3 corresponds to the upper edge of the field of view (slice $N$ ). 


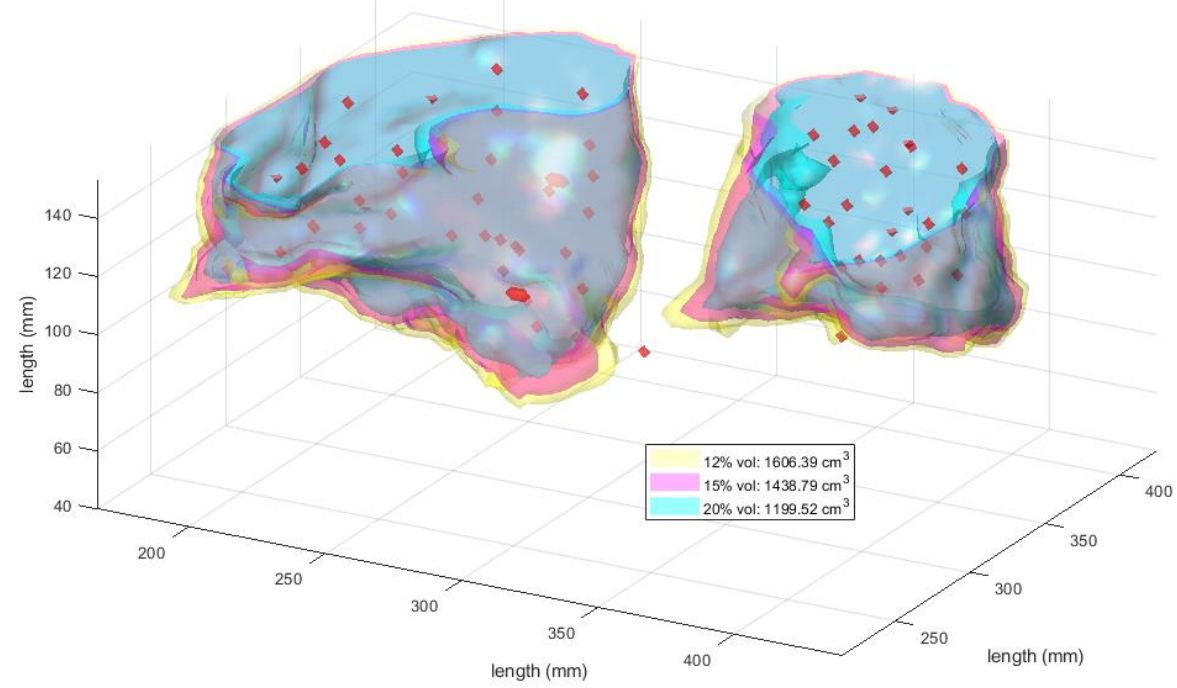

Fig.3. 3D image of reconstructed SPECT. Iso-surfaces for three different thresholds: $12 \%, 15 \%$ and $20 \%$ of the maximum count rate voxel are shown. The threshold $12 \%$ corresponds to the segmented volume in Figure 2. Red points are local maxima count rate voxels.

\section{Lung absorbed doses}

The lung absorbed dose was the amount of dose absorbed by the lungs. Estimated through the lung shunt fraction as follow:

The lung dose $D_{\text {lung }}$ was given by $[5,7]$ :

$$
D_{\text {lung }}=50 \times \frac{A_{\text {vial }} \times(1-R)}{M_{\text {lung }}} \times L S F \quad 6 .
$$

Dlung was the absorbed dose delivered to the lungs in Gy

$A_{\text {vial }}$ was the total Y-90 vial calibrated activity in GBq

$R$ was the residual fraction in empty syringe and other solid wastes generated in the infusion.

$L S F$ was the lung shunt fraction obtained above

50 was the dose in Gy delivered to a kg of mass by $1 \mathrm{GBq}$ of ${ }^{90} \mathrm{Y}[5]$

Mlung was the lung mass in $\mathrm{kg}$, assumed to be $1 \mathrm{~kg}$

$A_{\text {vial }}$ was determined for each treatment with a dose criteria [5, 7] from either tumor or perfused volumes. For example, using criteria for perfused volume, if the waste was negligible, the absorbed dose for this region: 


$$
D_{\text {per }}=50 \times \frac{A_{\text {per }}}{V_{\text {per }} \times 1.03}
$$

Where $V_{p e r}$ was the perfused volume (1), $A_{p e r}$ the perfused activity (GBq) and 1.03 was the liver tissue density $(\mathrm{kg} / \mathrm{l})$.

And:

$$
A_{\text {vial }}=\frac{A_{\text {per }}}{(1-L S F)}
$$

Combining 6, 7 and 8 results:

$$
D_{\text {lung }}=50 \times \frac{A_{\text {per }} \times L S F}{M_{\text {lung }} \times(1-L S F)}
$$

Therefore, the absorbed dose in lung depends on the LSF according to the rational equation:

$$
f=\frac{L S F}{(1-L S F)}
$$

10.

\section{RESULTS}

\section{A. LSF}

\begin{tabular}{|c|c|c|c|c|c|c|c|}
\hline \multicolumn{6}{|c|}{ STATIC PLANAR } & \multirow{2}{*}{\multicolumn{2}{|c|}{ SPECT CT }} \\
\hline \multicolumn{2}{|c|}{ AP } & \multicolumn{2}{|c|}{ PA } & \multicolumn{2}{|c|}{ TOTAL } & & \\
\hline & C & & C & & C & & \\
\hline Left lung & 243358 & Left lung & 330850 & Left lung & 574208 & & \\
\hline Right lung & 288278 & Right lung & 308058 & Right lung & 596336 & $\mathrm{c}(\mathrm{c} / \mathrm{ml})$ & 1193 \\
\hline \multirow[t]{2}{*}{ Liver } & 3573373 & Liver & 353879 & Liver & 3927252 & $M_{\text {lung }}(g)$ & 1000 \\
\hline & Cuentas & & Cuentas & & Cuentas & $V_{\text {lung }}(g)$ & 3333 \\
\hline Lung & 531636 & Lung & 638908 & Lung & 1427382 & $C_{\text {lung }}$ & 3977128 \\
\hline Total & 4105009 & Total & 992787 & Total & 5097796 & $C_{\text {total }}$ & 35628960 \\
\hline \multicolumn{4}{|c|}{ LSF } & \multicolumn{2}{|c|}{$28 \%$} & LSF & $11 \%$ \\
\hline
\end{tabular}

TABLE 1: The lung shunt fraction $L S F$ obtained from Method 1 (planar) and 2 (SPECT_CT) acquisitions performed for the studied patient. $C$ are the Counts within the indicated region 
In the studied case, the values $A_{\text {per }}$ was $1.26 \mathrm{GBq}$, and $A_{\text {vial }}$ planar was $1.75 \mathrm{GBq}$ and $A_{\text {vial }}$ spect was 1.42 then using equation 9, the absorbed dose with method 1 results 24.50 Gy and 7.92 Gy with method 2, respectively.

Although the quotient of the $L S F$ values according to method 1 or 2 was $0.28 / 0.11=$ 2.51 , the respective quotient between the lung absorbed dose $D_{\text {lung }}$ was 3.09 , due to the covariance of lung dose with $L S F$ (equation 10).

\section{DISCUSSION}

Presently, most hospitals have the capability of $\mathrm{TARE}^{90} \mathrm{Y}$ treatments for patients undergoing hepatic malignancies and the SPECT/CT imaging with $\mathrm{MAA}^{99 \mathrm{~m}} \mathrm{Tc}$ is a common practice for pre-treatment planning. It is the interest to develop methods to evaluate the shunt lung fraction (as the most frequent complication in these therapies), that can be easily applied in clinical practice. An approach used for LSF calculation is based on two AP-PA static planar images. This method has the disadvantage of not having corrections for scatter nor attenuation nor resolution recovery and consequently the counts estimation is not very accurate and over conservative. What's more this practice delays the main SPECT/CT study by 20 minutes increasing the patient discomfort. The other approach proposed here is taking advantage of SPECT/CT imaging, acquired for pre-treatment planning, to evaluate $L S F$.

Determination of activity and volumes from nuclear medicine images is severely limited by spatial resolution, scatter and penetrations components, noise and the partial volume effect [15]. Using SPECT-CT modality image has several advantages, is corrected by scatter, attenuation and resolution recovery, and the total administered activity is directly proportional to the total counts in the acquisition. Nevertheless the static planar image do not support these corrections. All things considered, planar image not take into account the attenuation between the liver and the skin, which is a very important factor for low energy radionuclides imaging, (140 keV for $\left.{ }^{99 \mathrm{M}} \mathrm{Tc}\right)$. The differences between consider or not consider the attenuation in quantification can become a factor 2-4.

Ideally, to evaluate the lung shunt, two beds should be acquired, thus covering the entire anatomical region that includes lungs and liver. This requires twice the acquisition time for the patient ( 2000 seconds instead of $\sim 1000)$, that is not acceptable for patient comfort.

In this study to solve the problem that not all lung counts are included in the bed liver image, an experimentally confirmed hypothesis [1] is used: the concentration of activity in the lung area is very homogeneous. The only physiological disparity could be a higher, slight concentration in the lung bases, especially in the standing position, because they present a greater relative perfusion; as well as the vertices it presents a greater ventilation with respect to the perfusion they receive. 
The activity concentration is a magnitude directly proportional to the absorbed dose and its use allows estimate this dose in the reconstructed SPECT study, if volume of a given region is known, and as long as it is isolated from the rest of the areas active, such as the case of the pulmonary shunt. The procedure is robust in the sense that does not rely on the site border blurring reflected in the low-resolution SPECT images, and can be easily implemented on clinical work and applied to all regions of all patients, given the uniformity of those regions.

The activity concentration estimations is affected by uncertainties in the calibration mainly resulting from the variability of the activities and volumes. Ideally, an arterial vascular phantom could be built in order to achieve a similar geometry and activity distribution in clinic media. For future studies, it is of interest to investigate these different sources of uncertainty in detail.

Absorbed doses in lung is critical and, although the critical dose is not known in NM internal therapy procedures, in external radiotherapy it is known that 30 Gy increase pulmonary complications and is usually adopted as the critical dose also in internal dosimetry. As can be seen in results section, the SPECT-based method get a much lower value ( $8 \mathrm{~Gy}$ ) than that estimated from the static scan planar images (24 Gy) and, in fact, this result for the patient studied, with the planar method, may compromise the application of the future treatment with ${ }^{90}$ Ytrio.

\section{CONCLUSIONS}

In this study projected ${ }^{90} \mathrm{Y} L S F$ and lung absorbed dose $D_{\text {lung }}$ based in planar static and SPECT/CT ${ }^{99 \mathrm{M}} \mathrm{Tc}$ images of one treatment patient, have been described and compared.

The results show a dependence between the absorbed dose in the lung with the LSF according to a rational function $f=L S F /(1-L S F)$.

The values of $L S F$ and $D_{\text {lung }}$ obtained with PLANAR method are 2.54 and 3.15 higher than the values obtained with SPECT method, respectively. This is due to differences in the quantification method, especially to the absence of attenuation correction in the planar method. In the $L S F$ values, the difference is direct, but in the case of the $D_{\text {lung }}$ values the difference is greater due to covariant dependence of dose with activity and LSF.

Practically for the future, the SPECT-CT method is better and will be applied in new patients. 


\section{COMPLIANCE WITH ETHICAL STANDARDS}

\section{CONCFLICT OF INTERES}

Author Raquel Barquero declares that she has no conflict of interest. Author Javier Velasco declares that he has no conflict of interest. Author Antonio Hurtado declares that he has no conflict of interest. Author Berta Perez declares that she has no conflict of interest. Author Ricardo Ruano declares that he has no conflict of interest.

This study does not contain any studies with animals performed by any of the authors.

\section{ETHICAL APPROVAL}

All procedures performed in the studies involving humans participants were in accordance with the ethical standards of the institutional research committee and with the 1964 Helsinki declaration and its later amendments or comparable ethical standards.

This article does not contain any studies with animals performed by any of the authors.

Informed consent was obtained from all the individual participants included in the study.

\section{REFERENCES}

\footnotetext{
${ }^{1}$ Saini A, Wallace A., Alzubaidi S. et al. History and Evolution of Yttrium-90 Radioembolization for Hepatocellular Carcinoma. J Clin Med 2019; 8, 55

2 Miscalculated Lung Shunt Fraction for Planning of Hepatic Radioembolization, Caskey J.S.,, Kay M.D., McMillan N.A.,et al, Journal of Nuclear Medicine Technology volumen 48 Número 2 Páginas 184-186 junio de 2020.

3 Comparison of Tc-99m MAA Planar Versus SPECT/CT Imaging for Lung Shunt Fraction Evaluation Prior to Y-90 Radioembolization: Are We Overestimating Lung Shunt Fraction?, Elsayed M.Cheng B.Xing M. et al, Cardiovascular and interventional radiology volumen 44 Número 2 Páginas 254260 01/10/2020.

4 Prospective Study of Quantitative SPECT/CT for Evaluation of Lung Shunt Fraction Before SIRT of Liver Tumors. Dittmann H.[2], Kopp D.[3], Kupferschlaeger J.[3], et al Journal of nuclear medicine: official publication, Society of Nuclear Medicine volumen 59 Número 9 Páginas 1366-1372 septiembre de 2018.

${ }^{5}$ Therasphere training, University-Hospital Essen, $1^{\text {st }}$ October 2015

${ }^{6}$ Capintec CRC ${ }^{\mathrm{R}}-15 \mathrm{R}$, Capintec, Inc., Ramsey, NJ. USA.

${ }^{7}$ Mirada Simplicity ${ }^{90}$ Y 2.4.0, January 2021

${ }^{8}$ SYMBYA collimator data sheet

9 Image J 1.52p Rasband W S Image Processing and Analysis in Java (Bethesda, MD: US National Institutes of Health) (http://imagej.nih.gov/ij/).

${ }^{10}$ Zeintl J, Hans Viga A, Yahli A, Hornegger J and Kuwert T. Quantitative accuracy of clinical 99MTc SPECT/CT using ordered-subset expectation maximization with 3-dimensional resolution recovery, attenuation, and scatter correction, J Nucl Med 2010; 51:921-928. DOI: 10.2967/jnumed.109.071571.

11 ICRP 110, Adult reference computational phantoms, International Commission on Radological Protection and International Commission of Radiation Units and Measurements, 2009.
} 
${ }^{12}$ Nobuyuki Kanematsu, Relationship between mass density, electron density, and elemental composition of body tissues for Monte Carlo simulation in radiation treatment planning, Physics in Medicine and Biology 61(13) 5037-5050, 2016.

${ }^{13}$ M. Rodríguez-Frailea, M. Inarrairaegui. Radioembolization with 90Y-microspheres for liver tumors Radioembolización de tumores hepáticos con 90Y-microesferas. Rev Esp Med Nucl Imagen Mol. 2015;34(4):244-257.

${ }^{14}$ MATLAB

15 Yuni K. Dewaraja, Eric C. Frey, George Sgouros, MIRD 23, MIRD Pamphlet No. 23: Quantitative SPECT for Patient-Specific 3-Dimensional Dosimetry in Internal Radionuclide Therapy, J Nucl Med 2012; 53:1310-1325. 


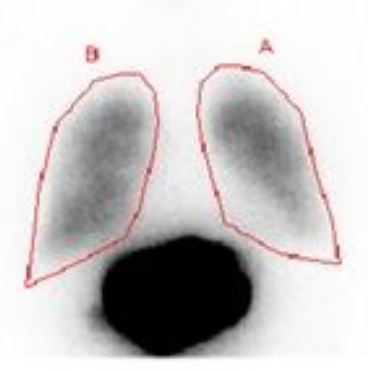

Anterior Torax $4377 \mathrm{~K}$ Conte os Duración.600\$ $256 \times 256$

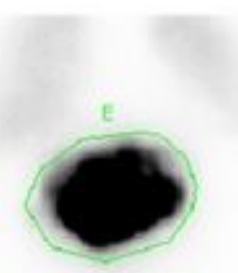

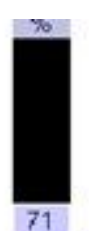
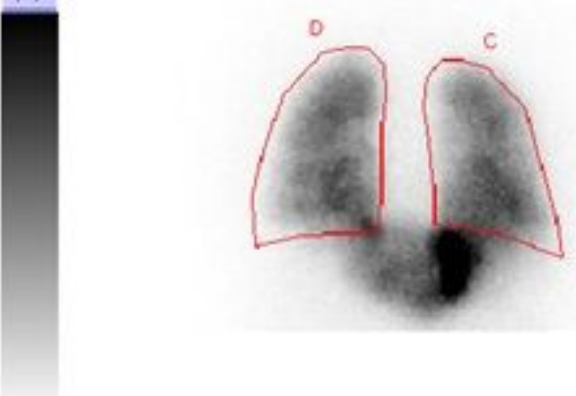

a

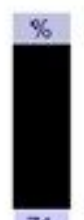

71

Posterior Toras 1146K Conteos Duración:600s 256x256

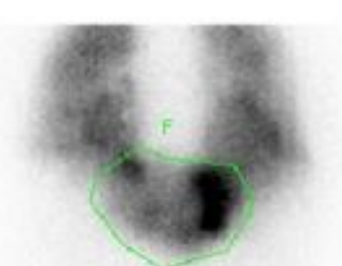

\section{Figure 1}

ROI of lung and liver of one patient. Images show, from top to bottom, lung ROIS and liver ROIS. From right to left the $0^{\circ}$ image and $180^{\circ}$ image, respectively. Two static acquisitions are needed to complete the lungs and the liver as a whole regions. 


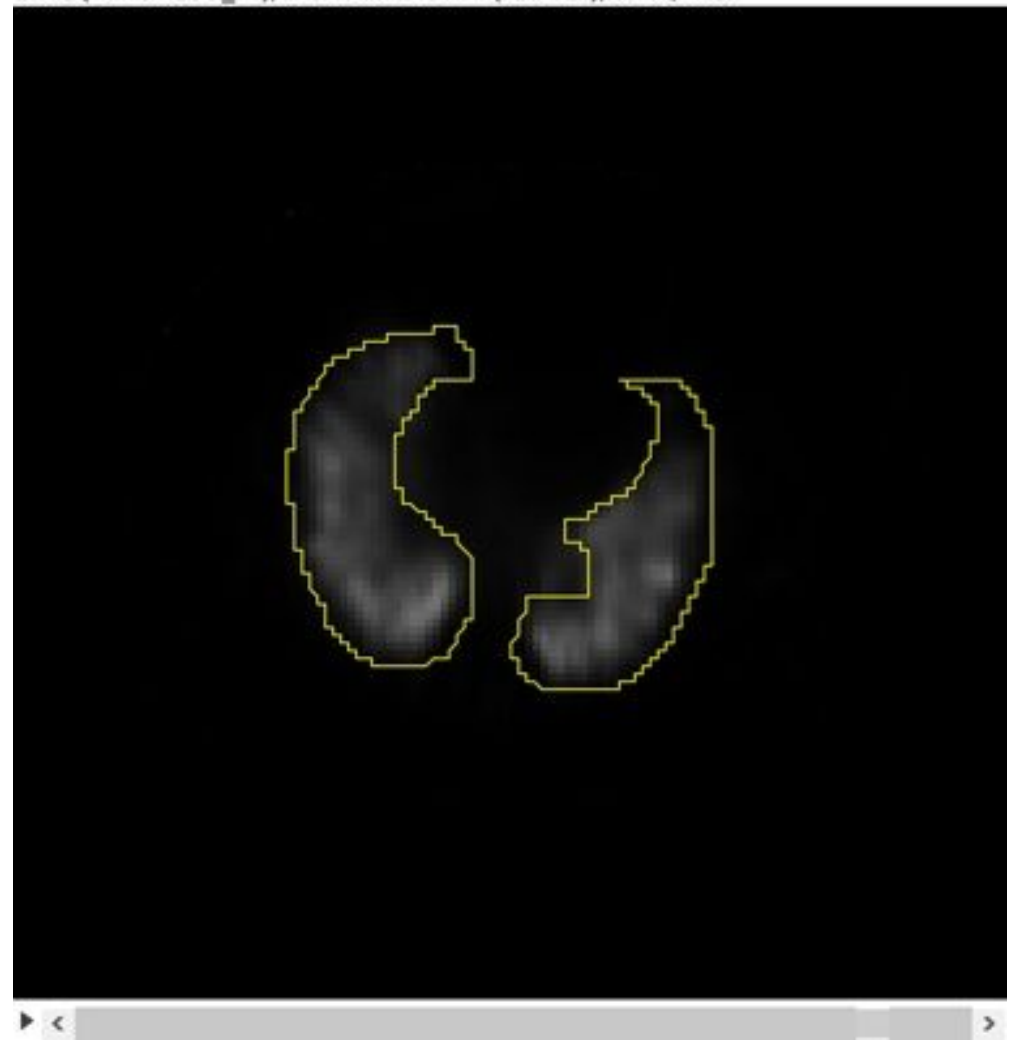

\section{Figure 2}

Reconstructed TRANSAXIAL SPECT image of one slice for one patient (LEHR collimation, $4.7295 \mathrm{~mm}$ voxel, $30 \mathrm{~ms}$ actual frame duration, 32 frames in rotation, OSEM 8i4s). VOls were drawn manually using CT boundaries as reference.

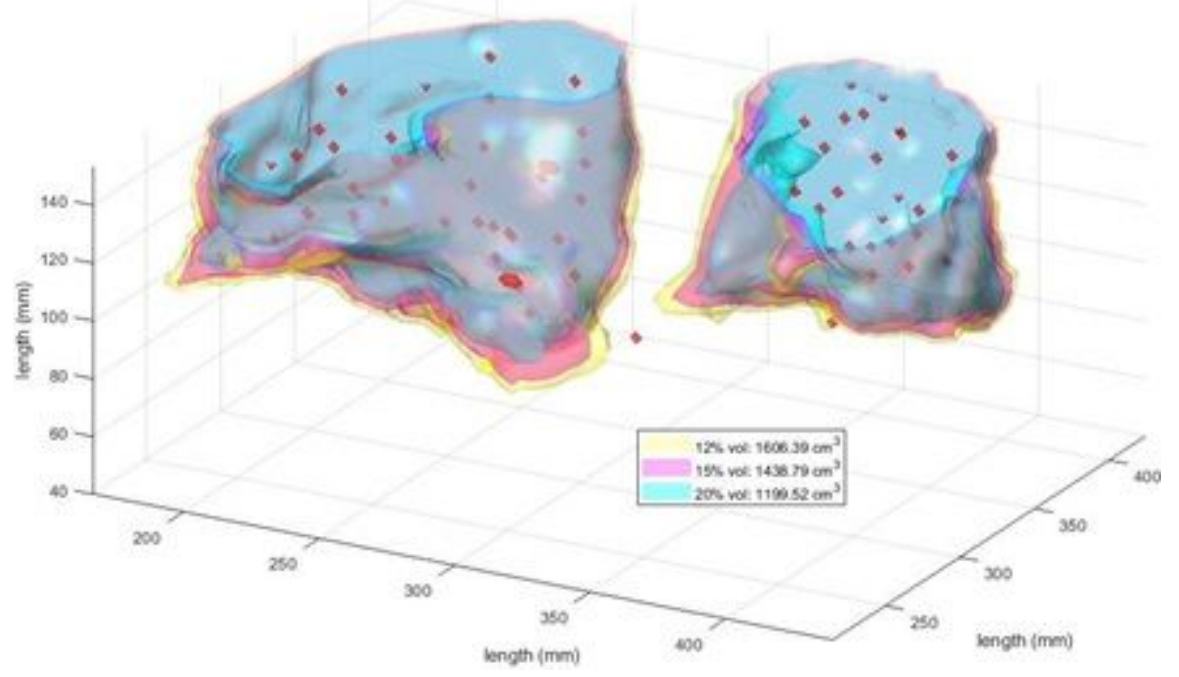

\section{Figure 3}

3D image of reconstructed SPECT. Iso-surfaces for three different thresholds: $12 \%, 15 \%$ and $20 \%$ of the maximum count rate voxel are shown. The threshold $12 \%$ corresponds to the segmented volume in Figure 
2. Red points are local maxima count rate voxels. 